BRX TH-549

\title{
Why is the metric invertible?
}

\author{
S. Deser \\ Department of Physics, Brandeis University, Waltham, MA 02454 \\ and \\ Lauritsen Laboratory, California Institute of Technology, Pasadena, CA 91125
}

\begin{abstract}
We raise, and provide an (unsatisfactory) answer to, the title's question: why, unlike all other fields, does the gravitational "metric" variable not have zero vacuum? After formulating, without begging it, we exhibit additions to the conventional action that express existence of the inverse through a field equation.

The metric variable's invertibility is (on a par with its dimensionality and signature) a tacit, but basic, assumption of gravitational theories. Unlike all other fields' dynamical variables, it does not vanish in the ground (or any other) state. This property is taken for granted (but see [1] for a recent attempted explanation) because it underlies existence of geometry and because the background-independence of covariant models does not single out any natural "zero". Nevertheless, even if spacetime is but an emergent property of some substrate, one should still seek an intrinsic explanation of "why there is something rather than nothing". Ours will exhibit additions to gravitational actions that embody invertibility as a field equation. The result will be far less satisfactory (or at least less familiar) than the Higgs effect's construction of a non-vanishing VEV.

The first difficulty is just to establish a framework where invertibility is not presupposed; we invoke the Palatini, first-order, approach where metric and affinity are independent variables. For concreteness, consider ordinary GR in $\mathrm{D}=4$,

$$
L_{E}=g^{\mu \nu} R_{\mu \nu}(\Gamma) .
$$

Here $g^{\mu \nu}$ is a symmetric contravariant density, $\Gamma_{\mu \nu}^{\alpha}$ is likewise $(\mu \nu)$ symmetric, and $R_{\mu \nu}(\Gamma)$ is the usual affine Ricci tensor constructed from $\Gamma$; note that $($ at $\mathrm{D}=4) \sqrt{-\operatorname{det} g^{\mu \nu}}$ is a scalar density.
\end{abstract}

The Palatini procedure requires solving the field equation

$$
D_{\alpha}(\Gamma) g^{\mu \nu} \sim \partial_{\alpha} g^{\mu \nu}+g \Gamma=0
$$

for $\Gamma(g)$. This is where invertibility of $g^{\mu \nu}$ comes in: without it, $\Gamma$ remains undetermined. Second order, Hilbert-Einstein, actions where $\Gamma$ is already the metric affinity obviously cannot even be written, absent invertibility.

Having pinpointed the requirement's origin, we provide the most elementary formal enlargement of the action (1) so that invertibility becomes a consequence of the field equations. The simplest way to ensure that $g^{\mu \nu}$ is nonsingular is of course that its determinant not 
vanish. [In this connection, we emphasize that our task is obtaining the inequality $\sqrt{-g}>0$, rather than imposing (as in [1]) the condition $\sqrt{-g}=1$.] To this end, we append the term

$$
L_{1}=M(\sqrt{-g} \sqrt{-h}-1)
$$

to $L_{E}$. We have been forced to introduce a new (covariant anti-density) tensor field, $h_{\mu \nu}$, although a simple scalar anti-density $h$ would have sufficed. With either choice, one finds that $\sqrt{-g} \sqrt{-h}=1$, as desired to guarantee invertibility. Then varying $h$ implies that $M=0$, since $\sqrt{-g} \neq 0$. The basic mechanism of (3) can be made more elaborate in

$$
L_{2}=M_{\mu}^{\nu}\left(g^{\mu \alpha} h_{\alpha \nu}-\delta_{\nu}^{\mu}\right)
$$

Varying the mixed tensor $M_{\nu}^{\mu}$ ensures that $g^{\mu \nu}$ has an inverse, while the contributions from $g$ - and $h$-variations vanish. Once we have a "normal" metric at hand, we can use it to move indices as usual. We then learn that the symmetric part of $M_{\mu \nu}$ - the only part that enters in the field equations - vanishes. A final variant is the pseudo-Higgs

$$
L_{3}=\tilde{M}\left(\sqrt{g h}-\phi^{2}\right)
$$

where the Higgs scalar $\phi$ has a non-zero VEV. This is, however, a rather empty "improvement" since one can absorb the $\phi^{2}$ into the density $\tilde{M}$ and the metrics. None of these ansätze provide any physical insight into the origin of the invertibility, even though they accomplish our formal aim: the Lagrange multipliers remain proxies for a worthier explanation.

It may be instructive to contrast the above background-free framework to that of the self-interaction bootstrap of [2] in which free tensor gauge field $\left(h^{\mu \nu}, \Gamma_{\mu \nu}^{\alpha}\right)$ propagates on a fixed but arbitrary background geometry, with an invertible metric $g_{0}^{\mu \nu}$ and its associated connection $\gamma_{\mu \nu}^{\alpha}\left(g_{0}\right)$. Its action is just the linearization of (1) about $g_{0}^{\mu \nu}$, with $g=g_{0}+h$. For consistency of the resulting field equations, the background is required to be Ricci-flat: $R_{\mu \nu}\left(\gamma\left(g_{0}\right)\right)=0$; the $\Gamma(h)$ relation (2) becomes perfectly soluble,

$$
D_{\alpha} h^{\mu \nu}=\left(g_{0} \Gamma\right)_{\alpha}^{\mu \nu} \Rightarrow \Gamma=g_{0}^{-1} D h
$$

where $D_{\alpha}$ is the background covariant derivative. The full Einstein theory for $g=g_{0}+h$ is simply recovered upon restoring the cubic $L_{3} \sim h \Gamma \Gamma$ term, modifying (6) to

$$
D_{\alpha} h^{\mu \nu}=\left[\left(g_{0}+h\right) \Gamma\right]_{\alpha}^{\mu \nu}
$$

For generic $h^{\mu \nu}$, the invertibility of $\left(g_{0}^{\mu \nu}+h^{\mu \nu}\right)$ is protected by that of $g_{0}^{\mu \nu}$. In our previous language, this procedure consists in a by-hand separation of the metric variable into a sum, one term of which is simply declared to define a fixed, invertible, background geometry: the question is still begged, if less obviously.

I thank R. Jackiw and A. Schwimmer for very patient discussions. This work was supported in part by NSF grant PHY04-01667. 


\section{References}

[1] F. Wilczek, Phys. Rev. Lett. 80, 4851 (1998).

[2] S. Deser, Class. Quant. Grav. 4, L99 (1987). 